\title{
Dimer-atom scattering between two identical fermions and a third particle
}

\author{
M. Iskin \\ Department of Physics, Koç University, Rumelifeneri Yolu, TR-34450 Sariyer, Istanbul, Turkey
}

(Received 8 March 2010; published 27 April 2010)

\begin{abstract}
We use the diagrammatic $T$-matrix approach to analyze the three-body scattering problem between two identical fermions and a third particle (which could be a different species of fermion or a boson). We calculate the $s$-wave dimer-atom scattering length for all mass ratios, and our results exactly match the results of Petrov. In particular, we list the exact dimer-atom scattering lengths for all available two-species Fermi-Fermi and Bose-Fermi mixtures. In addition, unlike that of the equal-mass-particles case where the three-body scattering $T$ matrix decays monotonically as a function of the outgoing momentum, we show that, after an initial rapid drop, this function changes sign and becomes negative at large momenta and then decays slowly to zero when the mass ratio of the fermions to the third particle is higher than a critical value (around 6.5). As the mass ratio gets higher, modulations of the $T$ matrix become more apparent with multiple sign changes, related to the "fall of a particle to the center" phenomenon and to the emergence of three-body Efimov bound states.
\end{abstract}

DOI: 10.1103/PhysRevA.81.043634

PACS number(s): 03.75.Ss, 03.75.Hh, 05.30.Fk

\section{INTRODUCTION}

The dimer-atom scattering process was first solved by Skorniakov and Ter-Martirosian in 1956 [1], in the context of three-nucleon scattering, that is, in which a two-body bound state between a neutron and a proton (called a deuteron) is scattering with a neutron. They considered equal-mass particles, and they found that while the Born approximation gives $a_{D A}=8 a_{A B} / 3$ for the scattering length between an $A B$ dimer and an $A$ atom, where $a_{A B}$ is the two-body scattering length between $A$ and $B$ particles, the exact result turns out to be $a_{D A} \approx 1.18 a_{A B}$ [1]. This problem has recently been analyzed with the diagrammatic $T$-matrix approach [2,3], in the context of cold quantum gases (see also [4] in a different context), and the result is in perfect agreement with the earlier results. In the same context, the three-body scattering problem has also been generalized to unequal-mass particles and analyzed both in real space through solving the three-body Schrödinger equation [5] and in momentum space through the diagrammatic $T$-matrix approach [6], with perfect agreement in between for all mass ratios.

When the mass ratio of the heavy particles to the light one is below 13.61, it is also well established that the three-body scattering problem is universal and that the dimer-atom scattering length is proportional to the two-body scattering length $a_{A B}$ (just like the equal-mass case) with the proportionality factor depending only on the masses of the constituent particles [5]. However, since three-body Efimov bound states emerge for larger mass ratios [7-9], this problem is not universal, and an additional parameter coming from the short-range (or large-momentum) three-body physics is needed for an accurate description.

Here, we use the diagrammatic $T$-matrix approach to analyze the three-body scattering problem between two identical fermions and a third particle. Our results are relevant to the quantum phases of two-species Fermi-Fermi [10-15] and Bose-Fermi [16-19] mixtures of atomic gases in the molecular limit. The paper is organized as follows. After deriving the Skorniakov and Ter-Martirosian integral equation generalized for unequal-mass particles in Sec. II A, we numerically solve the resultant equation for all mass ratios in Sec. II B, and we list the exact dimer-atom scattering lengths for all available two-species Fermi-Fermi and Bose-Fermi mixtures in Sec. IIC. A brief summary of our conclusions is given in Sec. III.

\section{THREE-BODY PROBLEM}

In this paper, we are interested in the three-body (dimeratom) $s$-wave scattering between two identical fermions (referred to as $A$-type particles) and a third particle (referred to as a $B$-type particle, which could be a different species of fermion or a boson). In particular, we consider a zero-range attractive interaction between $A$ and $B$ particles, and we assume there is a weakly bound resonance between them with the binding energy $\epsilon_{b}<0$, so that we want to study the scattering between this bound state (referred to as an $A B$ dimer) and the remaining $A$ fermion.

\section{A. Dimer-atom scattering $T$ matrix}

A detailed description of the diagrammatic $T$-matrix approach for the dimer-atom scattering process can be found in the literature for equal-mass particles [2-4], and here we give details of our calculation [6] for the case of unequal-mass particles.

We begin our analysis by describing the zero-temperature diagrammatic representation for the dimer-atom scattering $T$ matrix $T_{\mathbf{k}}^{D A}\left(\mathbf{p}, p_{0}\right)$ as illustrated in Fig. 1 , where $\mathbf{k}$ and $\mathbf{p}$ are the incoming and outgoing momenta, and $\omega_{A}=k^{2} /\left(2 m_{A}\right)$ and $\omega_{D}=k^{2} /\left(2 m_{D}\right)$ are the frequencies for the incoming $A$ particle and $A B$ dimer, respectively. Here, we set the center-of-mass momentum of the dimer-atom system to zero, and $\epsilon=\omega_{A}+\omega_{B}+\epsilon_{b}$ is the total frequency, where $\epsilon_{b}=$ $-1 /\left(m_{A B} a_{A B}^{2}\right)<0$ is the binding energy of the two-body bound state between $A$ and $B$ particles, and $m_{D}=m_{A}+$ $m_{B}$ and $m_{A B}=2 m_{B} m_{A} / m_{D}$ are masses of the $A B$ dimer and twice the reduced mass of the $A$ and $B$ particles, respectively. 


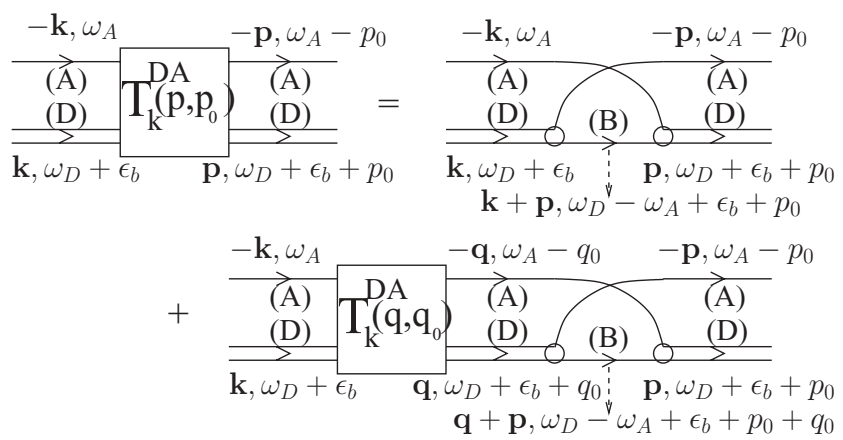

FIG. 1. Diagrammatic representation of the integral equation for the three-body scattering $T$ matrix $T_{\mathbf{k}}^{D A}\left(\mathbf{p}, p_{0}\right)$ between an $A B$ dimer (with dimer $D$ consisting of one fermionic particle $A$ and a second particle $B$ ) and a fermionic particle $A$. Particle $B$ could be a different species of fermion or a boson.

In Fig. 1, single lines represent retarded free propagators for the $A$ and $B$ particles, given by

$$
G_{A, B}(\mathbf{k}, \omega)=\frac{1}{\omega-\omega_{A, B}+\mu_{A, B}+i 0^{+}},
$$

where $\omega_{A, B}=k^{2} /\left(2 m_{A, B}\right)$ is the energy, and $\mu_{A, B}$ is the chemical potential of the corresponding particle. Similarly, double lines represent the retarded propagator for the $A B$ dimer, and in three dimensions it can be approximated by a simple one-pole structure:

$$
G_{D}(\mathbf{k}, \omega)=\frac{\frac{4 \pi}{m_{A B}}}{\frac{1}{a_{A B}}-\left[m_{A B}\left(\omega_{D}-\omega-\mu_{A}-\mu_{B}-i 0^{+}\right)\right]^{1 / 2}},
$$

which reflects the presence of a two-body bound state between $A$ and $B$ particles.

This dimer propagator is obtained from the resummation of the $A B$ polarization bubbles, leading to $G_{D}(\mathbf{k}, \omega)=$ $-g /\left[1+g \Gamma_{A B}(\mathbf{k}, \omega)\right]$, where $g>0$ is the strength of the bare interaction between $A$ and $B$ particles, and the $A B$ polarization bubble is $\Gamma_{A B}(\mathbf{k}, \omega)=\sum_{\mathbf{q}, q_{0}} G_{A}\left(\mathbf{k}+\mathbf{q}, \omega+q_{0}\right) G_{B}(-\mathbf{q}$, $\left.-q_{0}\right)$. At zero temperature, $\sum_{\mathbf{q}, q_{0}} \equiv i \int d \mathbf{q} d q_{0} /(2 \pi)^{4}$ in three dimensions. Integration over the internal momentum $\mathbf{q}$ and frequency $q_{0}$ leads to $\Gamma_{A B}(\mathbf{k}, \omega)=\Gamma_{A B}(\mathbf{0}, 0)+m_{A B}^{3 / 2}\left(\omega_{D}-\right.$ $\left.\omega-\mu_{A}-\mu_{B}-i 0^{+}\right)^{1 / 2} /(4 \pi)$, which in combination with the definition of the two-body scattering length $a_{A B}=$ $m_{A B} T^{A B}(\mathbf{0}, 0) /(4 \pi)$, and the two-body scattering $T$ matrix $T^{A B}(\mathbf{0}, 0)=-g /\left[1+g \Gamma_{A B}(\mathbf{0}, 0)\right]$, between $A$ and $B$ particles, give the final result described in Eq. (2).

In the following, we set $\mu_{A}=\mu_{B}=0$ since we are interested in the dimer-atom scattering in vacuum. However, note that our diagrammatic calculation for the scattering parameters of the three-body problem is exact, and they are sufficient to describe ultracold quantum gases, since experiments are always performed at low densities. The calculation of scattering parameters in the presence of many other particles (arbitrary density) is much more difficult, and it is not discussed here. In addition, on the right-hand side of Fig. 1, note that the first diagram represents a fermion exchange process (i.e., first particle $B$ breaks up with particle $A$ and then it forms a new $A B$ dimer with the remaining $A$ particle). This is the simplest process contributing to dimer-atom scattering (e.g., Born approximation), and all other (infinitely many) possible processes are included in the second diagram.

In analytical form, the dimer-atom $T$ matrix $T_{\mathbf{k}}^{D A}\left(\mathbf{p}, p_{0}\right)$ satisfies the following integral equation:

$$
\begin{aligned}
T_{\mathbf{k}}^{D A} & \left(\mathbf{p}, p_{0}\right) \\
= & -G_{B}\left(\mathbf{k}+\mathbf{p}, \omega_{D}-\omega_{A}+\epsilon_{b}+p_{0}\right) \\
& -\sum_{\mathbf{q}, q_{0}} G_{D}\left(\mathbf{q}, \omega_{D}+\epsilon_{b}+q_{0}\right) G_{A}\left(-\mathbf{q}, \omega_{A}-q_{0}\right) T_{\mathbf{k}}^{D A}\left(\mathbf{q}, q_{0}\right) \\
& \times G_{B}\left(\mathbf{p}+\mathbf{q}, \omega_{D}-\omega_{A}+\epsilon_{b}+p_{0}+q_{0}\right) .
\end{aligned}
$$

The minus signs on the right-hand side is due to Fermi-Dirac statistics (i.e., exchanging a fermion brings a minus sign unlike that of a boson). The integration over frequency $q_{0}$ can be easily performed by closing the integration contour in the upper half-plane, where both $T_{\mathbf{k}}^{D A}\left(\mathbf{q}, q_{0}\right)$ and $G_{D}\left(\mathbf{q}, \omega_{D}+\right.$ $\left.\epsilon_{b}+q_{0}\right)$ are analytic functions of $q_{0}$, and only a simple pole contribution comes from $G_{A}\left(-\mathbf{q}, \omega_{A}-q_{0}\right)$. Note that this property of $T_{\mathbf{k}}^{D A}\left(\mathbf{q}, q_{0}\right)$ is due to the form of Eq. (3) itself. This integration sets $q_{0}=\left(k^{2}-q^{2}\right) /\left(2 m_{A}\right)$, and we set $p_{0}=\left(k^{2}-p^{2}\right) /\left(2 m_{A}\right)$ in order to have the same frequency dependence for the $T$ matrix on both sides of Eq. (3). This leads to

$$
T_{\mathbf{k}}^{D A}(\mathbf{p})=\frac{m_{A B}}{p^{2}+\frac{m_{A B}}{m_{B}} \mathbf{p} \cdot \mathbf{k}+k^{2}-m_{A B} \epsilon}+\sum_{\mathbf{q}} \frac{4 \pi T_{\mathbf{k}}^{D A}(\mathbf{q})}{\left(q^{2}+\frac{m_{A B}}{m_{B}} \mathbf{p} \cdot \mathbf{q}+p^{2}-m_{A B} \epsilon\right)\left[\frac{1}{a_{A B}}-\left(\frac{m_{A B}}{m_{D A}} q^{2}-m_{A B} \epsilon\right)^{1 / 2}\right]},
$$

where we redefine the $T$ matrix $T_{\mathbf{k}}^{D A}(\mathbf{p})=T_{\mathbf{k}}^{D A}\left[\mathbf{p},\left(k^{2}-\right.\right.$ $\left.\left.p^{2}\right) /\left(2 m_{A}\right)\right]$, and $\epsilon=k^{2} / m_{D A}+\epsilon_{b}$ is the total energy and $m_{D A}=2 m_{D} m_{A} /\left(m_{D}+m_{A}\right)$ is twice the reduced mass of an $A B$ dimer and an $A$ particle. Since we are interested in zero-range low-energy $s$-wave scattering, we first average out directions of the incoming momentum $\mathbf{k}$, and then of the outgoing momentum $\mathbf{p}$, leading to

$$
\begin{aligned}
T_{k}^{D A}(p)= & \frac{m_{B}}{2 p k} \ln \left(\frac{p^{2}+\frac{m_{A B}}{m_{B}} p k+k^{2}-m_{A B} \epsilon}{p^{2}-\frac{m_{A B}}{m_{B}} p k+k^{2}-m_{A B} \epsilon}\right) \\
& +\int_{0}^{\infty} \frac{d q \frac{m_{B}}{m_{A B}} \frac{q}{\pi p} T_{k}^{D A}(q)}{\frac{1}{a_{A B}}-\left(\frac{m_{A B}}{m_{D A}} q^{2}-m_{A B} \epsilon\right)^{1 / 2}} \\
& \times \ln \left(\frac{q^{2}+\frac{m_{A B}}{m_{B}} p q+p^{2}-m_{A B} \epsilon}{q^{2}-\frac{m_{A B}}{m_{B}} p q+p^{2}-m_{A B} \epsilon}\right),
\end{aligned}
$$


for three-dimensional systems, where $T_{k}^{D A}(p)=\int d \Omega_{\mathbf{p}}$ $\int d \Omega_{\mathbf{k}} T_{\mathbf{k}}^{D A}(\mathbf{p}) /(4 \pi)^{2}$ is the angular-averaged $T$ matrix.

To obtain the three-body (dimer-atom) $s$-wave scattering length $a_{D A}$, the total energy $\epsilon$ should be set to the binding energy $\epsilon_{b}$ of the two-body bound state, in the limit of vanishing incoming and outgoing momentum and frequency [i.e., $\left.a_{D A}=2 m_{D A} T_{0}^{D A}(0) / m_{A B}^{2}\right]$. This motivates us to introduce the dimer-atom scattering function $a_{k}^{D A}(p)$ for which the integral equation becomes

$$
\begin{aligned}
\frac{\frac{m_{A B}}{m_{D A}} a_{0}^{D A}(p)}{\frac{1}{a_{A B}}+\left(\frac{m_{A B}}{m_{D A}} p^{2}+\frac{1}{a_{A B}^{2}}\right)^{1 / 2}} & \frac{1}{p^{2}+\frac{1}{a_{A B}^{2}}}-\frac{m_{B}}{\pi p m_{A B}} \int_{0}^{\infty} \frac{d q}{q} \\
& \times \ln \left(\frac{q^{2}+\frac{m_{A B}}{m_{B}} q p+p^{2}+\frac{1}{a_{A B}^{2}}}{q^{2}-\frac{m_{A B}}{m_{B}} q p+p^{2}+\frac{1}{a_{A B}^{2}}}\right) a_{0}^{D A}(q),
\end{aligned}
$$

in the limit when $k \rightarrow 0$. Here, the dimer-atom scattering length is $a_{D A}=a_{0}^{D A}(0)$, where

$$
a_{k}^{D A}(p)=\frac{m_{D A}}{m_{A B}^{2}}\left[\frac{1}{a_{A B}}+\left(\frac{m_{A B}}{m_{D A}} p^{2}-m_{A B} \epsilon\right)^{1 / 2}\right] T_{k}^{D A}(p)
$$

gives the full momentum dependence of the dimer-atom scattering function.

The integral equation shown in Eq. (6) and the scattering function expression shown in Eq. (7) reduce to the known results for the equal-mass-particles case [2,3] when $m_{A}=m_{B}=m$. Since only the fermion exchange process is taken into account in the Born approximation, neglecting the second term on the right-hand side of Eq. (6) leads to $a_{D A}=\left(2 m_{D A} / m_{A B}\right) a_{A B}$, which is consistent with the many-body results [20]. However, we need to include both terms and solve the integral equation in order to find the exact dimer-atom scattering length.

\section{B. Dimer-atom scattering function}

Next, we solve numerically the integral equation given in Eq. (6) as a function of the mass ratio $m_{A} / m_{B}$ of the constituent particles of the dimer [21]. For this purpose, it is convenient to change the upper integration limit to a finite value by a change of variables: $p a_{A B}=(1-x) /(1+x)$ and $q a_{A B}=(1-y) /(1+y)$, where $1 \geqslant\{x, y\} \geqslant-1$. The resultant integral is calculated by using the Gaussian-Legendre quadrature method, and using this discretization, we reduce the integral equation to a matrix-eigenvalue problem.

The exact solutions and the Born approximation values of $a_{D A}=a_{0}^{D A}(0)$ are shown in Fig. 2. When $m_{A}=m_{B}$, we find $a_{D A} \approx 1.18 a_{A B}$, which is in agreement with the results previously found for equal-mass particles [1-3,5]. The scattering length $a_{D A}$ increases (decreases) from this value with increasing (decreasing) mass ratio, and $a_{D A} \rightarrow a_{A B}$ in the limit of $m_{A} / m_{B} \rightarrow 0$ as expected. These results exactly match the few-body results of Petrov [5]. It is quite remarkable that the diagrammatic $T$-matrix approach exactly recovers the few-body results for all mass ratios, since the diagrammatic

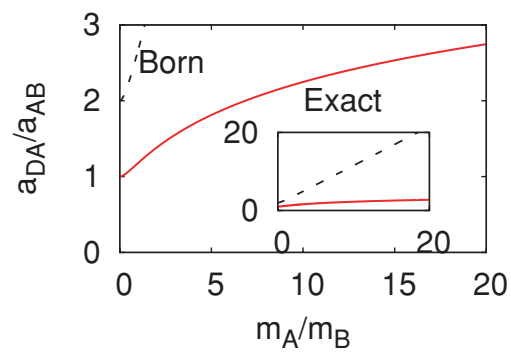

FIG. 2. (Color online) The exact scattering length $a_{D A}=a_{0}^{D A}(0)$ between an $A B$ dimer (with dimer $D$ consisting of one fermionic particle $A$ and a second particle $B$ ) and a fermionic particle $A$ as a function of mass ratio $m_{A} / m_{B}$ (solid red line) [21]. Particle $B$ could be a different species of fermion or a boson. Note that the disagreement between the exact value and the Born approximation one (dashed line) increases rapidly as the mass ratio increases as shown in the inset.

approach is performed in momentum space, while the fewbody approach is performed in real space [22]. Note that the Born approximation values for $a_{D A}$ are not in agreement with the exact values for any mass ratio and that the disagreement increases rapidly with increasing mass ratio, but the general qualitative trend is captured by the Born approximation as can be seen in the inset of Fig. 2.

In Fig. 3, we show the scattering function $a_{0}^{D A}(p)$ as a function of the outgoing momentum $p$ for some mass ratios $m_{A} / m_{B}$. When $m_{A}=m_{B}$, this is a monotonically decreasing (positive) function of $p$ with a long tail. However, beyond some critical mass ratio, this behavior changes

(a)

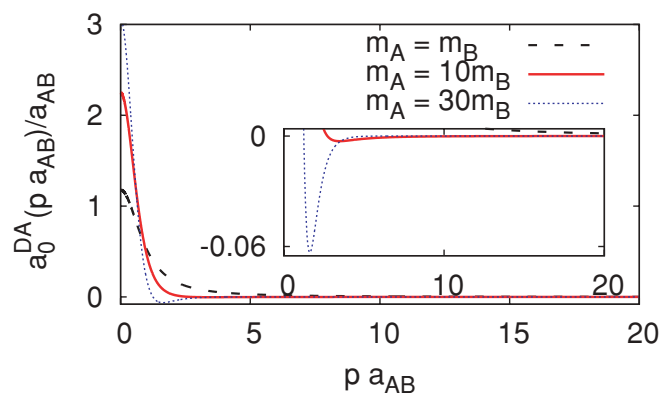

(b)

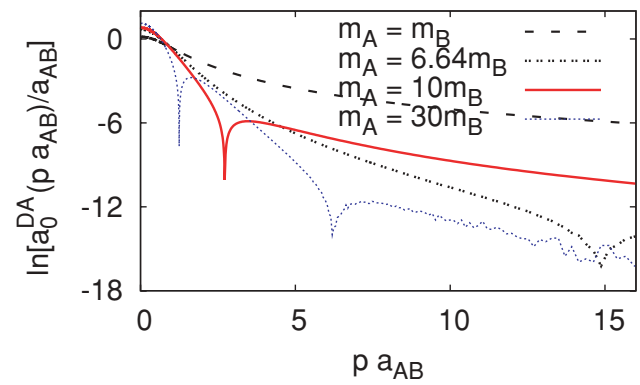

FIG. 3. (Color online) The scattering function $a_{0}^{D A}(p)$ between an $A B$ dimer (with dimer $D$ consisting of one fermionic particle $A$ and a second particle $B$ ) and a fermionic particle $A$ as a function of the outgoing momentum $p$ for some mass ratios $m_{A} / m_{B}$ [21]. Particle $B$ could be a different species of fermion or a boson. Here, all length scales are in units of the two-body scattering length between $A$ and $B$ particles, $a_{A B}$, and in (b) we show $\ln \left[a_{0}^{D A}(p)\right]$. 
dramatically. For instance, when $m_{A}=10 m_{B}$, after an initial rapid drop, the $a_{0}^{D A}(p)$ function changes sign and becomes negative at $p a_{A B} \approx 2.72$. Beyond this critical momentum, it reaches a minimum value and slowly decays to zero from the negative side. As the mass ratio gets lower (higher), $a_{0}^{D A}(p)$ changes sign at higher (lower) momenta. For instance, when $m_{A}=6.64 m_{B}$, corresponding to a three-body system consisting of one ${ }^{6} \mathrm{Li}$ atom and two ${ }^{40} \mathrm{~K}$ atoms, it changes sign at least once at $p a_{A B} \approx 14.7$ (but it changes sign at least once at $p a_{A B} \approx 1.96$ when $\left.m_{A}=13.61 m_{B}\right)$. We find clear sign changes when $m_{A} \gtrsim 6.5 m_{B}$, but we do not know whether $a_{0}^{D A}(p)$ changes sign for even lower mass ratios since precision issues obscure the results.

Although our calculation is not reliable for mass ratios above 13.61 [21], for illustration purposes, in Fig. 3(b), we show $\ln \left[a_{0}^{D A}(p)\right]$ as a function of $p$ when $m_{A}=30 m_{B}$. In this case, the scattering function first changes sign and becomes negative at $p a_{A B} \approx 1.25$, but then it changes sign again and becomes positive at $p a_{A B} \approx 6.2$. Since the large- $p$ behavior of this function is again obscured by precision issues, as can be seen in the figure, we could not resolve whether it has more sign changes at larger $p$ values. However, we mention that as the mass ratio increases further to $100, a_{0}^{D A}(p)$ changes sign at least four times, and the oscillating pattern becomes apparent.

This effect could be related to the "fall of a particle to the center" phenomenon $[9,23]$ and to the emergence of three-body Efimov bound states $[7,8]$, which are known to occur when the mass ratio is large. When the heavy $A$ particles are separated from each other by a distance $r \ll a_{A B}$ (or equivalently $\left.p a_{A B} \gg 1\right)$, it is known that an exchange of a light $B$ particle mediates an effective $-C /\left(m_{A} r^{2}\right)$ attraction between $A$ particles. Here, the coefficient $C \approx 0.162 m_{A} / m_{B}$ increases with increasing mass ratio [7]. Since $A$ particles are fermions, this attraction competes with the Pauli repulsion, which manifests itself as a centrifugal $\ell(\ell+1) /\left(m_{A} r^{2}\right)$ potential, where $\ell$ is the angular momentum. Therefore, when the masses are comparable $(C \approx 0.162)$, the Pauli repulsion is about one order of magnitude stronger than the mediated attraction. When the mass ratio $m_{A} / m_{B}$ reaches a critical value [i.e., when $C=\ell(\ell+1)]$, the effective interaction (mediated attraction plus centrifugal repulsion) between $A$ particles vanishes. For the lowest $p$-wave angular momentum channel $\ell=1$, this occurs when $m_{A} \gtrsim 12.33 m_{B}$.

Beyond this critical mass ratio, there is a second critical mass ratio beyond which the effective attraction between $A$ particles is strong enough that the $A$ particles stay in an infinitely small region around each other (i.e., the remaining $A$ particle falls to the center of the attraction [23]). This second critical mass ratio can be approximated by $\gamma_{c}=1 / 4=$ $C-\ell(\ell+1)$ [24]. For the lowest $p$-wave angular momentum channel $\ell=1$, this occurs when $m_{A} \gtrsim 13.85 m_{B}$. Note that, since the $B$ particle is already bound to one of the $A$ particles, "fall of a particle to the center" is related to emergence of three-body Efimov bound states. More accurate calculations show that the latter occurs when $m_{A} \gtrsim 13.61 m_{B}$ [8,9].

Having presented the diagrammatic $T$-matrix approach for the three-body ( $s$-wave) dimer-atom scattering, for all mass ratios $m_{A} / m_{B}$, next we discuss the relevance of our results to the quantum phases of two-species Fermi-Fermi and BoseFermi mixtures of atomic gases at ultracold temperatures.

\section{Ultracold Fermi-Fermi and Bose-Fermi mixtures}

It has been shown that [20,25] (see [26] for experimental confirmation), in the strong-attraction or molecular limit, two-species Fermi-Fermi mixtures with population imbalance can be well described by effective Bose-Fermi models, where fermion-fermion pairs behave as molecular bosons (dimers) and interact weakly with each other and with the remaining unpaired (excess) fermions. These simpler models only require accurate scattering lengths between two molecular bosons (dimer-dimer, i.e., $a_{D D}$ ) and between a molecular boson and an unpaired fermion (dimer-atom, i.e., $a_{D A}$ ). Note that the exact three- and four-body results in vacuum are sufficient to describe ultracold atomic mixtures in the molecular limit, since experiments are always performed at low densities. Several fermionic atoms $\left({ }^{6} \mathrm{Li},{ }^{40} \mathrm{~K},{ }^{87} \mathrm{Sr}\right.$ [27], and ${ }^{171} \mathrm{Yb}$ [28]) are currently being investigated, and experimental methods for studying two-species Fermi-Fermi mixtures are being developed in several groups (e.g., the ${ }^{6} \mathrm{Li}^{40} \mathrm{~K}$ mixture [10-15]). Thus, anticipating future experiments involving various other mixtures, in Table I, we list the exact dimer-atom scattering lengths for all possible mixtures.

Similarly, in the strong-attraction or molecular limit, two-species Bose-Fermi mixtures (with more fermions than bosons; otherwise see [29]) can be well described by effective Fermi-Fermi models, where boson-fermion pairs can be shown to behave as molecular fermions (dimers) and interact weakly with the remaining unpaired fermions. These simpler models again only require accurate scattering length between a molecular fermion and an unpaired fermion (dimer-atom, i.e., $\left.a_{D A}\right)$. Experimental methods for studying two-species Bose-Fermi mixtures are also being developed in several groups [16-19], and in Table II, we list the exact dimer-atom scattering lengths for all possible mixtures.

As was pointed out earlier, the three-body scattering problem is universal for mass ratios below 13.61, and therefore, the dimer-atom scattering is proportional to the two-body

TABLE I. The exact scattering length $a_{D A}=a_{0}^{D A}(0)$ between a bosonic $A B$ dimer (consisting of one fermionic $A$ atom and one fermionic $B$ atom) and a fermionic $A$ atom is shown for a list of twospecies Fermi-Fermi mixtures. (When $A$ and $B$ atoms have exactly the same mass, $A$ and $B$ labels refer to different spin states of the same species.) Here, $a_{D A}$ is in units of the two-body scattering length between $A$ and $B$ particles, $a_{A B}$, and the mass ratios $m_{A} / m_{B}$ are shown inside the parentheses [21].

\begin{tabular}{lccccc}
\hline \hline \multicolumn{5}{c}{$A$} \\
\cline { 2 - 6 }$B$ & ${ }^{6} \mathrm{Li}$ & ${ }^{40} \mathrm{~K}$ & ${ }^{87} \mathrm{Sr}$ & ${ }^{171} \mathrm{Yb}$ & ${ }^{173} \mathrm{Yb}$ \\
\hline${ }^{6} \mathrm{Li}$ & 1.17907 & 1.98106 & 2.50583 & 3.01531 & 3.02437 \\
& $(1.00000)$ & $(6.64392)$ & $(14.4484)$ & $(28.4178)$ & $(28.7506)$ \\
${ }^{40} \mathrm{~K}$ & 1.01033 & 1.17907 & 1.41148 & 1.72559 & 1.73187 \\
& $(0.15051)$ & $(1.00000)$ & $(2.17468)$ & $(4.27726)$ & $(4.32735)$ \\
${ }^{87} \mathrm{Sr}$ & 1.00251 & 1.06337 & 1.17907 & 1.37374 & 1.37799 \\
& $(0.06921)$ & $(0.45984)$ & $(1.00000)$ & $(1.96685)$ & $(1.98988)$ \\
${ }^{171} \mathrm{Yb}$ & 1.00069 & 1.02195 & 1.07331 & 1.17907 & 1.18159 \\
& $(0.03519)$ & $(0.23379)$ & $(0.50843)$ & $(1.00000)$ & $(1.01171)$ \\
${ }^{173} \mathrm{Yb}$ & 1.00067 & 1.02153 & 1.07209 & 1.17657 & 1.17907 \\
& $(0.03478)$ & $(0.23109)$ & $(0.50254)$ & $(0.98842)$ & $(1.00000)$ \\
\hline \hline
\end{tabular}


TABLE II. The exact scattering length $a_{D A}=a_{0}^{D A}(0)$ between a fermionic $A B$ dimer (consisting of one fermionic $A$ atom and one bosonic $B$ atom) and a fermionic $A$ atom is shown for a list of two-species Bose-Fermi mixtures. Here, $a_{D A}$ is in units of the two-body scattering length between $A$ and $B$ particles, $a_{A B}$, and the mass ratios $m_{A} / m_{B}$ are shown inside the parentheses [21].

\begin{tabular}{|c|c|c|c|c|c|}
\hline \multirow[b]{2}{*}{$B$} & \multicolumn{5}{|c|}{$A$} \\
\hline & ${ }^{6} \mathrm{Li}$ & ${ }^{40} \mathrm{~K}$ & ${ }^{87} \mathrm{Sr}$ & ${ }^{171} \mathrm{Yb}$ & ${ }^{173} \mathrm{Yb}$ \\
\hline \multirow[t]{2}{*}{${ }^{7} \mathrm{Li}$} & 1.14817 & 1.88773 & 2.39579 & 2.89621 & 2.90516 \\
\hline & (0.857 34) & $(5.69612)$ & (12.3872) & (24.3638) & $(24.6491)$ \\
\hline \multirow[t]{2}{*}{${ }^{23} \mathrm{Na}$} & 1.026642 & 1.33055 & 1.66074 & 2.05182 & 2.05926 \\
\hline & $(0.26164)$ & (1.738 34) & (3.780 33) & (7.435 32) & $(7.52240)$ \\
\hline \multirow[t]{2}{*}{${ }^{39} \mathrm{~K}$} & 1.01080 & 1.18459 & 1.42138 & 1.73929 & 1.74563 \\
\hline & (0.154 38) & (1.025 67) & (2.230 51) & (4.387 07) & $(4.43844)$ \\
\hline \multirow[t]{2}{*}{${ }^{41} \mathrm{~K}$} & 1.00988 & 1.17371 & 1.40181 & 1.71213 & 1.71835 \\
\hline & $(0.14677)$ & (0.975 16) & $(2.12067)$ & $(4.17103)$ & $(4.21988)$ \\
\hline \multirow[t]{2}{*}{${ }^{84} \mathrm{Sr}$} & 1.00268 & 1.06670 & 1.18675 & 1.38665 & 1.39100 \\
\hline & (0.071 68) & $(0.47625)$ & $(1.03570)$ & $(2.03706)$ & (2.060 91) \\
\hline \multirow[t]{2}{*}{${ }^{85} \mathrm{Rb}$} & 1.00262 & 1.06556 & 1.18413 & 1.38226 & 1.38658 \\
\hline & (0.070 84) & $(0.47065)$ & $(1.02352)$ & $(2.013$ 10) & $(2.03668)$ \\
\hline \multirow[t]{2}{*}{${ }^{86} \mathrm{Sr}$} & 1.00256 & 1.06445 & 1.18157 & 1.37796 & 1.38225 \\
\hline & $\left(\begin{array}{lll}0.070 & 02\end{array}\right)$ & (0.465 19) & (1.011 63) & (1.989 73) & $(2.01303)$ \\
\hline \multirow[t]{2}{*}{${ }^{87} \mathrm{Rb}$} & 1.00251 & 1.06337 & 1.17906 & 1.37373 & 1.37799 \\
\hline & $\left(\begin{array}{l}0.069 \\
21)\end{array}\right.$ & $(0.45984)$ & (0.999 99) & (1.966 84) & (1.989 87) \\
\hline \multirow[t]{2}{*}{${ }^{88} \mathrm{Sr}$} & 1.00245 & 1.06232 & 1.17662 & 1.36961 & 1.37382 \\
\hline & $(0.06843)$ & $(0.45462)$ & $(0.98866)$ & (1.944 54) & (1.967 32) \\
\hline \multirow[t]{2}{*}{${ }^{133} \mathrm{Cs}$} & 1.00112 & 1.03306 & 1.10408 & 1.23978 & 1.24291 \\
\hline & $(0.04526)$ & $(0.30069)$ & (0.653 91) & $(1.28615)$ & $(1.30121)$ \\
\hline \multirow{2}{*}{${ }^{135} \mathrm{Cs}$} & 1.00109 & 1.03228 & 1.10200 & 1.23581 & 1.23890 \\
\hline & $(0.04459)$ & (0.296 24) & $(0.64422)$ & $(1.26708)$ & $(1.28192)$ \\
\hline \multirow[t]{2}{*}{${ }^{168} \mathrm{Yb}$} & 1.00071 & 1.02260 & 1.07519 & 1.18292 & 1.18548 \\
\hline & $(0.03582)$ & (0.237 97) & $(0.51752)$ & (1.017 58) & $(1.02980)$ \\
\hline \multirow[t]{2}{*}{${ }^{170} \mathrm{Yb}$} & 1.00070 & 1.02217 & 1.07393 & 1.18034 & 1.18297 \\
\hline & $(0.03540)$ & (0.235 17) & $(0.51142)$ & (1.005 89) & (1.017 67) \\
\hline \multirow[t]{2}{*}{${ }^{172} \mathrm{Yb}$} & 1.00068 & 1.02174 & 1.07270 & 1.17781 & 1.18032 \\
\hline & (0.034 98) & $(0.23243)$ & (0.505 47) & (0.994 18) & (1.005 83) \\
\hline \multirow[t]{2}{*}{${ }^{174} \mathrm{Yb}$} & 1.00067 & 1.02132 & 1.07150 & 1.17534 & 1.17783 \\
\hline & (0.034 58) & $(0.22976)$ & $(0.49965)$ & (0.982 74) & $(0.99425)$ \\
\hline \multirow[t]{2}{*}{${ }^{176} \mathrm{Yb}$} & 1.00065 & 1.02092 & 1.07033 & 1.17292 & 1.17538 \\
\hline & (0.034 19) & $(0.22714)$ & $(0.49396)$ & $(0.97155)$ & $(0.98292)$ \\
\hline
\end{tabular}

scattering length $a_{A B}$ with the proportionality factor depending only on the masses of the constituent particles. However, since three-body Efimov bound states emerge for larger mass ratios $[7,8]$, this problem is not universal (with an additional parameter coming from the short-range three-body physics being needed for an accurate description), and our analysis does not include this nonuniversal effect [5,9]. In particular, the ${ }^{6} \mathrm{Li}^{8}{ }^{87} \mathrm{Sr},{ }^{6} \mathrm{Li}^{-171} \mathrm{Yb}$, and ${ }^{6} \mathrm{Li}^{173}{ }^{173} \mathrm{Yb}$ Fermi-Fermi mixtures and ${ }^{7} \mathrm{Li}^{-}{ }^{171} \mathrm{Yb}$, and ${ }^{7} \mathrm{Li}^{1}{ }^{173} \mathrm{Yb}$ Bose-Fermi mixtures have mass ratios that are above the critical ratio for the emergence of three-body Efimov bound states.

\section{CONCLUSIONS}

In this paper, we used the diagrammatic $T$-matrix approach to analyze the three-body scattering problem between two identical fermions and a third particle. The third particle could be a different species of fermion or a boson. We calculated the exact $s$-wave dimer-atom scattering length for all mass ratios, and our results exactly match the few-body results of Petrov who obtained them by solving the three-body Schrödinger equation [5]. It is quite remarkable that the diagrammatic $T$-matrix approach exactly recovers the few-body results for all mass ratios, since the diagrammatic approach is performed in momentum space, while the few-body approach is performed in real space [22].

We also discussed the relevance of our results to the quantum phases of two-species Fermi-Fermi and Bose-Fermi mixtures of atomic gases at ultracold temperatures. In particular, in the strong-attraction or molecular limit, these mixtures can be well described by simpler effective models, where paired atoms behave as dimers and interact weakly with each other and with the remaining unpaired atoms. These effective descriptions require only the scattering lengths between two dimers and between a dimer and an unpaired atom. Anticipating future experiments, we listed the exact dimer-atom scattering lengths for all available two-species Fermi-Fermi and Bose-Fermi mixtures.

In addition, we showed that, unlike that of the equal-massparticles case where the three-body scattering $T$ matrix decays monotonically as a function of the outgoing momentum, after an initial rapid drop, this function changes sign and becomes negative at large momenta and then decays slowly to zero when the mass ratio of the fermions to the third particle is higher than a critical value (around 6.5). As the mass ratio gets higher, modulations of the $T$ matrix become more apparent with multiple sign changes. We argued that this effect could be related to the "fall of a particle to the center" phenomenon $[9,23]$ and to the emergence of three-body Efimov bound states $[7,8]$.

\section{ACKNOWLEDGMENTS}

The author thanks P. Pieri for useful discussions and the Scientific and Technological Research Council of Turkey (TÜBITAK) for financial support.
[1] G. V. Skorniakov and K. A. Ter-Martirosian, Zh. Eksp. Teor. Fiz. 31, 775 (1956) [Sov. Phys. JETP 4, 648 (1957)].

[2] I. V. Brodsky, M. Yu. Kagan, A. V. Klaptsov, R. Combescot, and X. Leyronas, Phys. Rev. A 73, 032724 (2006).

[3] J. Levinsen and V. Gurarie, Phys. Rev. A 73, 053607 (2006).

[4] P. F. Bedaque and U. van Kolck, Phys. Lett. B 428, 221 (1998).
[5] D. S. Petrov, Phys. Rev. A 67, 010703(R) (2003).

[6] M. Iskin and C. A. R. Sá de Melo, Phys. Rev. A 77, 013625 (2008).

[7] V. N. Efimov, Yad. Fiz. 12, 1080 (1970) [Sov. J. Nucl. Phys. 12, 589 (1971)]; Nucl. Phys. A 210, 157 (1973).

[8] O. I. Kartavtsev and A. V. Malykh, J. Phys. B 40, 1429 (2007). 
[9] D. S. Petrov, C. Salomon, and G. V. Shlyapnikov, J. Phys. B 38, S645 (2005).

[10] M. Taglieber, A.-C. Voigt, T. Aoki, T. W. Hänsch, and K. Dieckmann, Phys. Rev. Lett. 100, 010401 (2008).

[11] E. Wille, F. M. Spiegelhalder, G. Kerner, D. Naik, A. Trenkwalder, G. Hendl, F. Schreck, R. Grimm, T. G. Tiecke, J. T. M. Walraven, S. J. J. M. F. Kokkelmans, E. Tiesinga, and P. S. Julienne, Phys. Rev. Lett. 100, 053201 (2008).

[12] A.-C. Voigt, M. Taglieber, L. Costa, T. Aoki, W. Wieser, T. W. Hänsch, and K. Dieckmann, Phys. Rev. Lett. 102, 020405 (2009).

[13] F. M. Spiegelhalder, A. Trenkwalder, D. Naik, G. Hendl, F. Schreck, and R. Grimm, Phys. Rev. Lett. 103, 223203 (2009).

[14] T. G. Tiecke, M. R. Goosen, A. Ludewig, S. D. Gensemer, S. Kraft, S. J. J. M. F. Kokkelmans, and J. T. M. Walraven, Phys. Rev. Lett. 104, 053202 (2010); J. Levinsen, T. G. Tiecke, J. T. M. Walraven, and D. S. Petrov, ibid. 103, 153202 (2009).

[15] F. M. Spiegelhalder et al., e-print arXiv:1001.5253 (2010).

[16] S. Inouye, J. Goldwin, M. L. Olsen, C. Ticknor, J. L. Bohn, and D. S. Jin, Phys. Rev. Lett. 93, 183201 (2004).

[17] S. Ospelkaus, C. Ospelkaus, L. Humbert, K. Sengstock, and K. Bongs, Phys. Rev. Lett. 97, 120403 (2006).

[18] M. Zaccanti, C. D’Errico, F. Ferlaino, G. Roati, M. Inguscio, and G. Modugno, Phys. Rev. A 74, 041605(R) (2006).
[19] J. J. Zirbel, K.-K. Ni, S. Ospelkaus, J. P. D’Incao, C. E. Wieman, J. Ye, and D. S. Jin, Phys. Rev. Lett. 100, 143201 (2008).

[20] M. Iskin and C. A. R. Sá de Melo, Phys. Rev. Lett. 97, 100404 (2006); Phys. Rev. A 76, 013601 (2007).

[21] Our results are accurate only for mass ratios that are smaller than the critical value 13.61, beyond which an additional parameter coming from the short-range (or large-momentum) three-body physics is needed for an accurate description [5,7-9], which is beyond the scope of this paper.

[22] The two methods are equivalent because all of the possible scattering processes are taken into account exactly in our diagrammatic approach at zero temperature.

[23] L. D. Landau and E. M. Lifshitz, Quantum Mechanics (Butterworth-Heinemann, Oxford, 1999), p. 114.

[24] See Eq. (35.2) and the discussion above Eq. (35.10) for the two-body scattering problem in [23].

[25] P. Pieri and G. C. Strinati, Phys. Rev. Lett. 96, 150404 (2006).

[26] Y.-I. Shin, A. Schirotzek, C. H. Schunck, and W. Ketterle, Phys. Rev. Lett. 101, 070404 (2008).

[27] R. Grimm (private communication).

[28] T. Fukuhara, Y. Takasu, M. Kumakura, and Y. Takahashi, Phys. Rev. Lett. 98, 030401 (2007).

[29] K. Helfrich, H.-W. Hammer, and D. S. Petrov, e-print arXiv:1001.4371 (2010). 